\title{
The Silence of Neurosyphilis
}

\section{Karim Richard Masri, M.D., Sylvia Orozco-Do, M.D. and Andrew Massey, M.D.}

\section{Introduction}

Meningosyphilis is an uncommon cause of stroke in the post-antibiotic era and is considered a disease of the past. With the advent of antibiotics, it is rarely encountered. We present a case of late-stage syphilis infection.

\section{Case}

A 54-year-old African-American male presented with bilateral pedal frostbite after a snow storm. He demonstrated loss of sensation in his lower extremities. He was confused and dysphasic and was unable to articulate his history coherently but did endorse abdominal pain, nausea and vomiting. Initial CT scanning of the head could not rule out a subacute infarct versus abscess in the left posterolateral temporal lobe. A comprehensive workup was unrevealing except for positive serum and CSF VDRL. Tabes dorsalis was suspected.

\section{Tabes dorsalis}

- 20 years post-infection

- Dorsal columns and dorsal roots affected

- Sensory abnormalities and/or lancinating pains affecting the face, back or limbs

- Paresthesias, absent lower extremity reflexes, depressed vibratory and position sensation, attenuated touch and pain

- Gastric crises which manifest as recurrent nausea, vomiting with severe epigastric pain

- Argyll-Robertson pupil is one of the most common presenting signs in tabes dorsalis but our patient did not display this manifestation

\section{References}

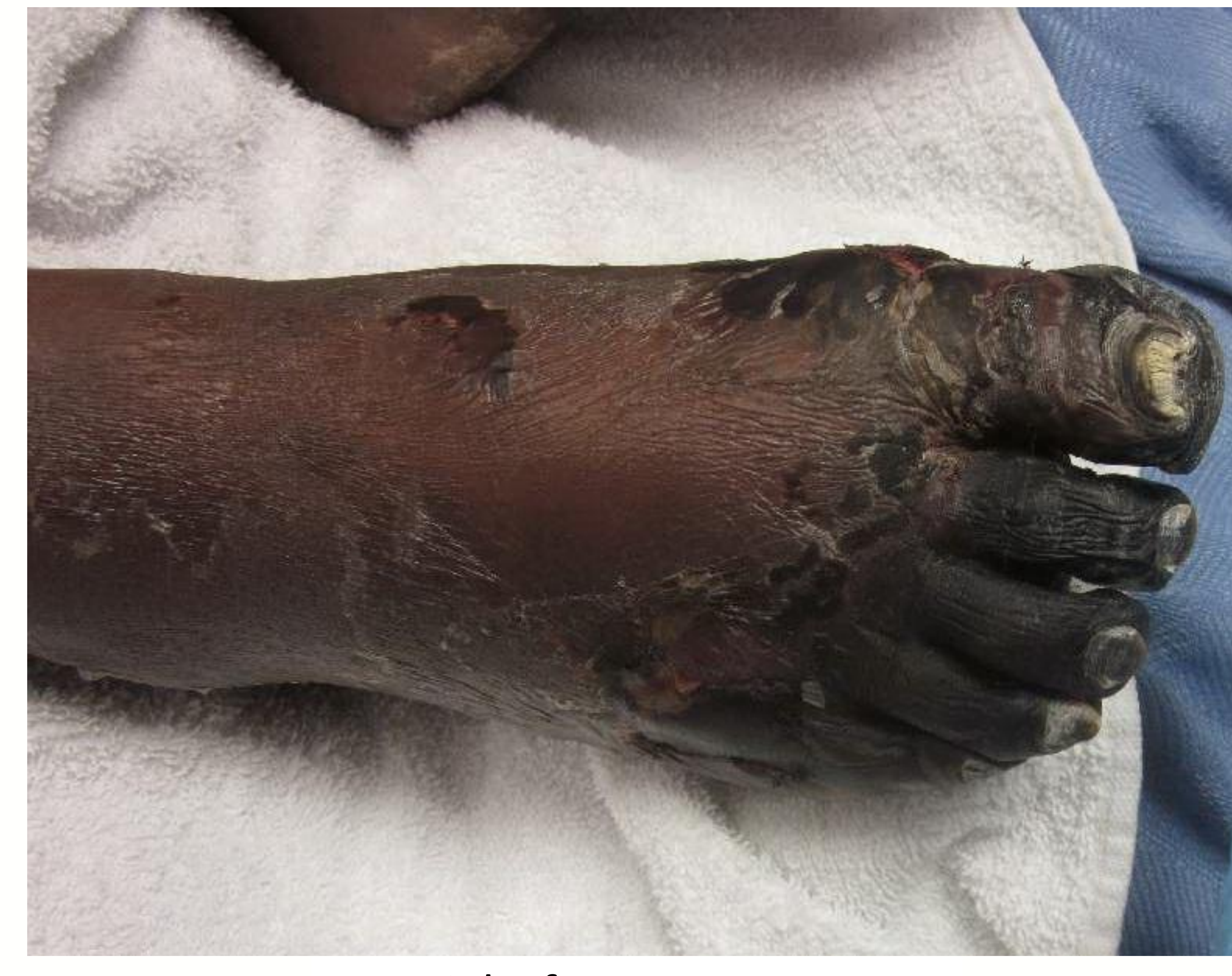

Right foot gangrene.

\section{Patient Follow Up}

The patient eventually underwent bilateral toe amputations and numerous debridement procedures and was placed in a nursing home for physical rehabilitation.

\section{Discussion}

Neurosyphilis is a known cause of central nervous system vasculitis with the potential for stroke. In patients who present with symptoms suggestive of a stroke, syphilis should always be considered in the differential diagnosis. Tabes dorsalis typically manifests with sensory deficits in the lower extremities and with gastric crises, both of which were present in our patient. Diagnosis of neurosyphilis is made with a CSFVDRL. A negative serum VDRL or RPR does not rule out CNS manifestations. Treatment for neurosyphilis, including tabes dorsalis, is $18-24$

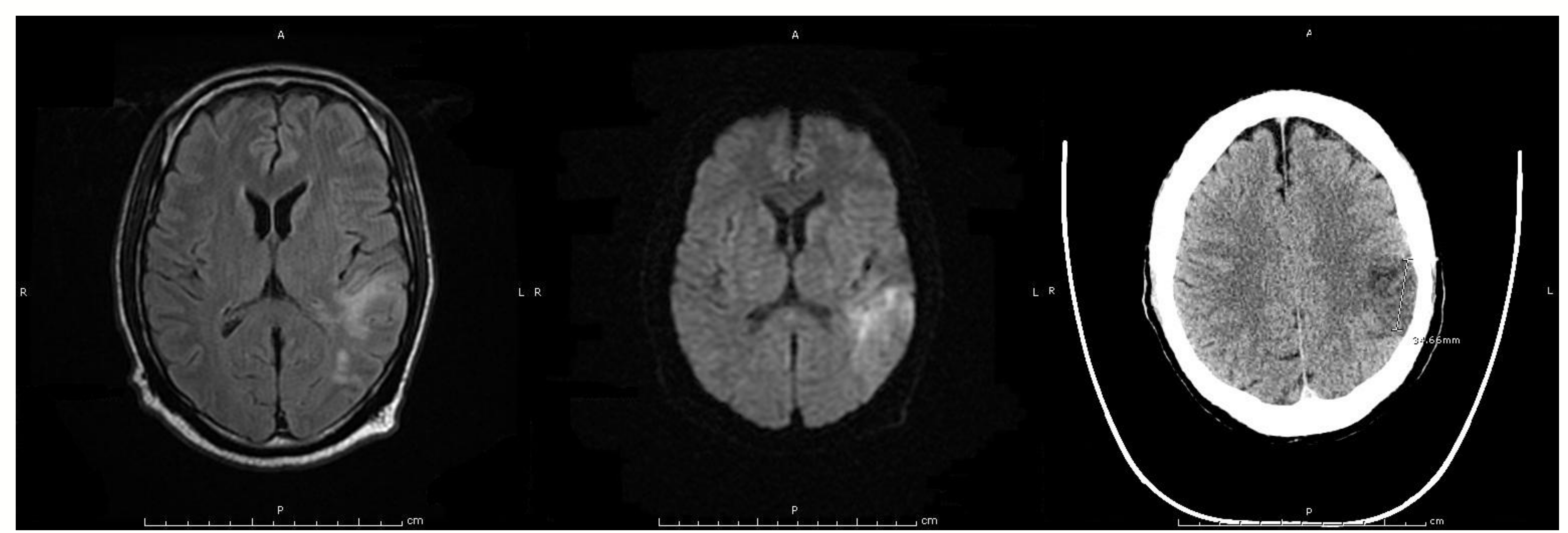

(LEFT) T2 Flair demonstrates high signal in the left temporal parietal region and (MIDDLE) the diffusion study reveals that the high signal on T2 Flair is due to cytotoxic edema. (RIGHT) Abnormal geographic region of decreased attenuation left parietal frontal lobe. This may be reflective of an area of acute-to-subacute non-hemorrhagic infarct.

Centers for Disease Control and Prevention. Atlanta, GA, 2010. Available from: http://www.cdc.gov/std/stats09/Syphilis.htm

Musher D. Early syphilis. In: Holmes, KK, Sparling PF. Mardh, PA, et al (Eds), Sexually Transmitted Diseases. New York, NY, McGraw-Hill, 1999, p.479.

Clark, EG, Danbolt, N. The Oslo study of the natural course of untreated syphilis: An epidemiologic investigation based on a re-study of the Boeck-Bruusgaard material. Med Clin North Am 1964; 48:613.

Pleimes M, Hartschuh W, Kutzner H, Enk AH, Hartmann M. Malignant syphilis with ocular involvement and organismdepleted lesions. Clin Infect Dis 2009;48(1):83-5.
Penicillin G infused daily for two weeks.

Early Neurosyphilis Symptoms and Complications

- Meningismus

- Altered mental status or confusion

- Audiovisual impairments

- Stroke

- Seizures

\section{Late Neurosyphilis}

Complications

- Stroke

- General paresis

- Tabes dorsalis 